\title{
Sanitätsdienstliche Führung im Grossereignis (SFG)
}

Mathias Zürchera , Stefan Müllerb, Pierre-Nicolas Carron ${ }^{c}$, Bruno Durrerd, Heinz Zimmermanne, Stefan Trachself , Wolfgang Ummenhoferg

a PD Dr. med., Leitender Arzt Rettungs- und Katastrophenmedizin, Leiter SFG, Universitätsspital Basel; b Dr. med., Facharzt Anästhesie, Notarzt SGNOR, Chefarzt Schutz \& Rettung Zürich; ' PD Dr. med., Médecin adjoint, Secteur préhospitalier, Service des Urgences, CHUV, Lausanne; ${ }^{d}$ Dr. med., Facharzt Allgemeine Innere Medizin, Sportmedizin SGSM, Notarzt SGNOR, Lauterbrunnen; ${ }^{e}$ Prof. Dr. med., Co-Präsident SGNOR, Bern; ${ }^{\dagger}$ Chef Geschäftsstelle KSD, Ittigen; ${ }^{g}$ Prof. Dr. med., Leitender Arzt Anästhesiologie, Universitätsspital Basel

In einer Artikelserie zum 2010 in der Schweizerischen Ärztezeitung erschienenen «8-Punkte-Leitbild der FMH zum Rettungswesen» vertieft und erläutert die Plattform Rettungswesen die einzelnen Aspekte des Leitbilds. Der folgende Artikel bezieht sich auf Punkt 7: Sanitätsdienstliche Führung im Grossereignis (SFG). Die verbindliche Koordination ist der Schlüssel zur erfolgreichen Kooperation und Bewältigung medizinischer Grossereignisse.

Grossereignisse und Katastrophen sind zum Glück selten. Trotzdem darf das Gefahrenpotential in unserem dicht besiedelten Land insbesondere entlang der Transitkorridore, die vielfach durch Ballungszentren führen, nicht vernachlässigt werden. Zudem können jederzeit störungsanfällige Infrastrukturen weitreichende Schäden anrichten.

Grossereignisse bringen auch grössere Rettungsdienste schnell an Kapazitätsgrenzen. Das kantonal und zum

\begin{tabular}{ll}
\hline Abkürzungen & \\
\hline ABC-Ereignis & Atomares, biologisches und chemisches Ereignis \\
\hline AHLS & Advanced Hazmat Life Support \\
\hline BABS & Bundesamt für Bevölkerungsschutz \\
\hline BL San & Bereichsleiter Sanität \\
\hline CEFOCA & Centre de formation en médecine de catastrophe \\
\hline CHUV & Centre Hospitalier Universitaire Vaudois \\
\hline CSAM & Conduite sanitaire en cas d'accident majeur \\
\hline EL San & Einsatzleiter Sanität \\
\hline GDK & $\begin{array}{l}\text { Konferenz der kantonalen Gesundheitsdirektorinnen } \\
\text { und -direktoren }\end{array}$ \\
\hline IES & Informations- und Einsatz-System \\
\hline IVR & Interverband für Rettungswesen \\
\hline Komp Zen SAMK & $\begin{array}{l}\text { Kompetenzzentrum für Militär- und Katastrophenmedizin; } \\
\text { vormals Schweizerische Akademie für Militär- und Katastrophen- } \\
\text { medizin }\end{array}$ \\
\hline KSD & Koordinierter Sanitätsdienst \\
\hline LNA & Leitender Notarzt \\
\hline PLS & Patientenleitsystem \\
\hline SFG & Sanitätsdienstliche Führung Grossereignis \\
\hline SGNOR & Schweizerische Gesellschaft für Notfall- und Rettungsmedizin \\
\hline USB & Universitätsspital Basel \\
\hline VRS & Vereinigung Rettungssanitäter Schweiz \\
\hline
\end{tabular}

Teil regional organisierte Gesundheits- und Rettungswesen erfordert deshalb eine effiziente und übergreifende Kooperation. Bereits 2004 haben der Koordinierte Sanitätsdienst (KSD) und die Schweizerische Gesellschaft für Notfall- und Rettungsmedizin (SGNOR) eine nationale Weiterbildung in «Sanitätsdienstlicher Führung Grossereignis» (SFG) etabliert.

Das Weiterbildungsprogramm SFG-CSAM wird auf Deutsch durch das Universitätsspital Basel (USB) als Kurs «Sanitätsdienstliche Führung Grossereignis» (SFG-P, präklinisch) sowie auf Französisch durch das Centre de formation en médecine de catastrophe (CEFOCA) am Centre Hospitalier Universitaire Vaudois (CHUV) als Kurs "Conduite sanitaire en cas d'accident majeur" (CSAM, modules M2 + M3) angeboten. Dabei werden Rettungssanitäter und Notärzte gemeinsam und interprofessionell für ihre jeweiligen Führungsfunktionen «Bereichsleiter Sanität» (BL San), «Einsatzleiter Sanität» (EL San) resp. "Leitender Notarzt» (LNA) weitergebildet (Details: www.cefoca-sfg.ch).

\section{Führung und Management im Fokus}

Die Weiterbildung SFG-CSAM fokussiert auf Führungsund Managementkompetenzen. Wissen und praktische Fähigkeiten werden im Rahmen der spezifischen Planspiele in kleinen Gruppen ergänzt, die als Trainingsgeräte für die Führungsausbildung dienen (Abb. 1: Führungspyramide SFG). Die Richtlinien für die Organisation des Sanitätsdienstes bei Ereignissen mit grossem Patientenanfall sowie zum Patientenleitsystem des Interver- 


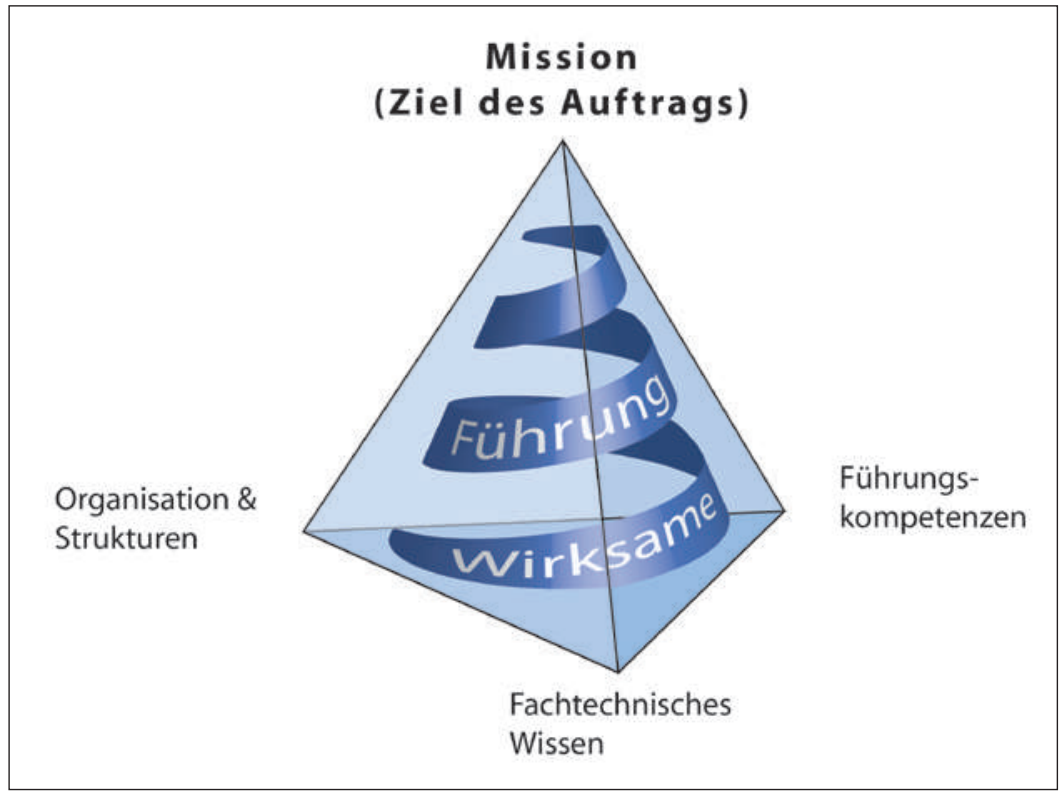

Abbildung 1: Führungspyramide SFG.

bands für Rettungswesen (IVR) werden berücksichtigt. In den meisten Kantonen ist die Weiterbildung SFG breit akzeptiert, in den grossen Rettungsdiensten umgesetzt und regional gut implementiert. Eine gesamtschweizerische Weiterbildung mit gemeinsamer Terminologie und einheitlichen Grundkonzepten hilft, dass die föderalistisch organisierten Rettungsdienste bei Grossereignissen optimal zusammenarbeiten.

\section{«Unité de doctrine SFG/CSAM» - ein Auf- trag der GDK realisiert durch den KSD}

2014 hat die Konferenz der kantonalen Gesundheitsdirektorinnen und -direktoren (GDK) dem KSD den Auftrag erteilt, zusammen mit der SGNOR, der Vereinigung Rettungssanitäter Schweiz (VRS) und weiteren Partnern eine «Unité de doctrine SFG/CSAM» für Lehrinhalte, Methodik, Qualität, Zertifizierung und Logistik zu definieren und umzusetzen.

2015 wurde von einer Kerngruppe des KSD zusammen mit den Weiterbildungspartnern am CHUV und am USB die «Doctrine SFG/CSAM» für die Weiter- und Fortbildung erarbeitet und durch einen breit abgestützten Konsensprozess validiert. Die auf der Doctrine basieren-

\section{Weiterführende Links:}

Informationen zu SFG und CSAM: www.cefoca-sfg.ch Koordinierter Sanitätsdienst (KSD): www.ksd-ssc.ch Terminologie Rettungs-, Notfall- und Katastrophenmedizin: www.rescuepoint.ch

Schweizerische Gesellschaft für Notfall- und Rettungsmedizin: www.sgnor.ch den Ausführungsbestimmungen sowie weitere Aspekte zur Organisation und Administration werden bis Ende 2016 folgen. Der KSD stärkt durch diese Koordination die Weiter- und Fortbildung SFG-CSAM und damit auch die Bedeutung der Diplome «EL San» resp. «LNA».

\section{Das Informations- und Einsatz-System (IES) des KSD}

Für die Bewältigung von Grossereignissen müssen neben einer effizienten Schadenplatzorganisation auch die Zielspitäler optimal mit allen prähospitalen Strukturen vernetzt werden. Diese Vernetzung wird durch das vom KSD entwickelte IT-System «Information und Einsatz» (IES) gewährleistet. Dieses unterstützt beim Grossereignis die Notrufeinsatzzentralen, die Schadenplatzorganisation sowie die Zielspitäler. Der zeitaktuelle Informationsaustausch ermöglicht beispielsweise eine überregionale, optimale Disposition der Transport-, Medikamenten-, Behandlungs- und Intensivbettenkapazitäten. Alle mit dem IES gewonnenen Erfahrungen werden auch hinsichtlich Benutzerfreundlichkeit evaluiert. Regelmässige Updates können zukünftig die Bewältigung der seltenen Grossereignisse weiter optimieren.

\section{Weitere Elemente zur Optimierung bei Grossereignissen}

Grossereignisse sind auch für die spitalinterne Organisation eine Ausnahme und eine grosse Herausforderung. SFG und CEFOCA bieten deshalb auch Weiterbildungen für das spitalinterne Management von Grossereignissen/Katastrophen an (Kurse SFG-H [hospital] / CEFOCA-M6).

Der KSD unterstützt zusätzlich Weiterbildungen zu Spezialthemen, die im Rahmen des Fortbildungsprogramms SFG-CSAM angeboten werden. Diese Spezialthemen umfassen:

- Medizinisches Management bei Ereignissen aus dem ABC-Bereich: Advanced-Hazmat-Life-Support-Kurs (AHLS)

- Medientraining Ereignisse im Rettungswesen (Kurs SFG-M)

- Weiterbildung für die Tätigkeit als Arzt in der Beratungsstelle Radioaktivität

- Ereignisse mit zahlreichen Schwerbrandverletzten (Swiss Burn Plan)

- Care (Angebote des Nationalen Netzwerks für Psychologische Nothilfe, NNPN)

Zudem stellt der KSD die notwendigen Plattformen zur Verfügung, um koordinierende Vorarbeiten leisten zu können. 


\section{Empfehlung für die Bewältigung grösserer sanitätsdienstlicher Ereignisse}

Alle kantonalen Verantwortungsträger sollten sich an den etablierten Weiterbildungen des KSD orientieren und dafür notwendige Ressourcen zur Verfügung stellen. Dank Kooperationen kann eine wirksame und kosteneffiziente Vorhalteleistung für diese speziellen Situationen erreicht werden.

\section{Kernsätze:}

Korrespondenz:

Prof. Dr. med.

Wolfgang Ummenhofer Departement Anästhesie Universitätsspital Basel CH-4031 Basel

wolfgang.ummenhofer[at] usb.ch
- Gemeinsam erarbeitete verbindliche Konzepte sind die Voraussetzung für eine erfolgreiche Kooperation verschiedener Organisationen.

- Um medizinische Grossereignisse wirklich zu bewältigen, müssen die nationalen Konzepte in Übungen auf ihre Tauglichkeit überprüft werden.
- Nur durch eine nationale Koordination kann auch im Grossereignis ein nahezu individualmedizinischer Versorgungsstandard erreicht werden.

\section{Literatur}

- Lupi GA. Sanitätsdienstliche Führung Grossereignis; KSD, 16.2.04.

- Interverband für Rettungswesen IVR. «Richtlinie zur Organisation des Sanitätsdienstes bei grossem Patientenanfall sowie bei besonderen und ausserordentlichen Lagen"; 2006.

- Interverband für Rettungswesen IVR. «Richtlinie für das Patientenleitsystem im Rettungswesen (PLS)»; 2003.

- KSD-Bulletin 4/2008: Schulung CEFOCA-SFG (Dies kann unter infoksd[at]vtg.admin.ch angefordert werden).

- Carron PN, Reigner P, Vallotton L, Clouet JG, Danzeisen C, Zuercher $\mathrm{M}$, Yersin B. Implementation of a concept for binomial medical command and control in Switzerland. Disasters Journal 2014;38: 434-50. doi: 10.1111/disa.12043

Bildnachweis

(c) SFG-Kurs Faculty 